www.nature.com/jhg

\title{
De novo 19q13.42 duplications involving NLRP gene cluster in a patient with systemic-onset juvenile idiopathic arthritis
}

\author{
Hiromi Tadaki ${ }^{1,2}$, Hirotomo Saitsu ${ }^{1}$, Akira Nishimura-Tadaki ${ }^{1}$, Tomoyuki Imagawa ${ }^{2}$, Masako Kikuchi ${ }^{2}$, \\ Ryoki Hara $^{2}$, Utako Kaneko ${ }^{2}$, Takayuki Kishi ${ }^{2}$, Takako Miyamae ${ }^{2}$, Noriko Miyake ${ }^{1}$, Hiroshi Doi ${ }^{1}$, \\ Yoshinori Tsurusaki ${ }^{1}$, Haruya Sakai ${ }^{1}$, Shumpei Yokota ${ }^{2}$ and Naomichi Matsumoto ${ }^{1}$
}

Systemic-onset juvenile idiopathic arthritis (s-JIA) is a rare inflammatory disease classified as a subtype of chronic childhood arthritis, manifested by spiking fever, erythematous skin rash, pericarditis and hepatosplenomegaly. The genetic background underlying s-JIA remains poorly understood. To detect disease-related copy number variations (CNVs), we performed singlenucleotide polymorphism array analysis in 50 patients with s-JIA. We detected many CNVs, but most of them were inherited from either of normal-phenotype parents. However, in one patient, we could identify two de novo microduplications at $19 \mathrm{q} 13.42$ with the size of 77 and $622 \mathrm{~kb}$, separated by a 109-kb segment of normal copy number. The duplications encompass NLRP family (NLRP2, NLRP9 and NLRP11) as well as IL11 and HSPBP1, all of which have an important role in inflammatory pathways. These genes may significantly contribute to the pathogenesis of s-JIA. Journal of Human Genetics (2011) 56, 343-347; doi:10.1038/jhg.2011.16; published online 17 February 2011

Keywords: arthritis; de novo; duplication; systemic-onset JIA

\section{INTRODUCTION}

Juvenile idiopathic arthritis (JIA) is a chronic rheumatic disease of childhood. Approximately $11 \%$ of JIA patients show systemic-onset JIA (s-JIA), ${ }^{1}$ which is clinically manifested by spiking fever, erythematous skin rash, pericarditis and hepatosplenomegaly in addition to arthritis. Abnormal innate immunity involving cytokines such as interleukin (IL)-1 and IL-6, neutrophils and monocytes/macrophages may play a major role in the pathogenesis of s-JIA. One of the major features of s-JIA is its progression to macrophage activation syndrome. On the basis of all these evidences, it is now generally accepted that s-JIA should be classified as an autoinflammatory syndrome rather than a classical autoimmune disease.

Two genetic factors, HLA and PTPN22, have been found as JIA susceptibility genes in multiple populations. ${ }^{2}$ For example, HLA-B27, HLA-DR1 and HLA-DR4 have been reported to increase risk for polyarticular JIA. ${ }^{3}$ However, such associations are seen mostly in polyarticular JIA, but not in s-JIA. Other genes including MIF, IL6, IL10 and TNF are reported to be associated with s-JIA in different populations and subtypes, ${ }^{4-8}$ although these genes account for only a small part of the total genetic contribution to JIA. Therefore, the genetic background underlying the s-JIA remains mostly undetermined.
We performed genome-wide copy number variations (CNVs) analysis in s-JIA patients. Two de novo microduplications at 19q13.42 encompassing 77 and $622 \mathrm{~kb}$ were identified in one patient by single-nucleotide polymorphism (SNP) array 6.0 and confirmed by other methods. The duplications encompass NLRP (Nucleotide-binding oligomeriztion domain, Leucine rich Repeat and Pyrin domain) family (NLRP2, NLRP9 and NLRP11), which have important roles in inflammatory processes as well as IL11, which was reported to correlate with arthritis severity in s-JIA patients. This is the first report of de novo CNVs in relation to s-JIA.

\section{MATERIALS AND METHODS}

Subjects

A total of 50 patients with s-JIA, which was refractory to conventional treatment and was treated with tocilizumab, were enrolled with informed consent based on the IRB-approved protocols at Yokohama City University Hospital. There were no family histories in each patient. Genomic DNA of peripheral blood leukocytes from all patients were isolated using DNA isolation systems (Quick Gene-800; Fujifilm, Tokyo, Japan).

Whole-genome SNP array and custom array analyses

To detect CNVs, two different SNP array platforms, the Genechip Human Mapping 250K array (Affymetrix, Santa Clara, CA, USA) (for 23 patients) and

${ }^{1}$ Department of Human Genetics, Yokohama City University Graduate School of Medicine, Yokohama, Japan and ${ }^{2}$ Department of Pediatrics, Yokohama City University Graduate School of Medicine, Yokohama, Japan

Correspondence: Professor N Matsumoto, Department of Human Genetics, Yokohama City University Graduate School of Medicine, 3-9 Fukuura, Kanazawa-ku, Yokohama 236-0004, Japan.

E-mail: naomat@yokohama-cu.ac.jp

Received 13 December 2010; revised 15 January 2011; accepted 17 January 2011; published online 17 February 2011 
the Genome-wide Human SNP array 6.0 (Affymetrix) (for the other 27 patients), were used following the manufacturer's protocols. In brief, for the Genome-wide Human SNP array 6.0, 500-ng DNA was digested with Nspl and Sty1 (only Nsp1 was used for $250 \mathrm{~K}$ array). The adaptors were ligated to the digested DNA, and the ligation-mediated polymerase chain reaction (PCR) with single primer was performed. PCR products were purified by magnetic beads (Ampure; Beckman Coulter Company, Beverly, MA, USA). Microcon-YM100 (Millipore Corporation, Bedford, MA, USA) was used for purification for the $250 \mathrm{~K}$ array. The product was fragmented, end-labeled and hybridized to an array. CNAG3.0 (ref. 9), Genotyping Console (Copy Number Analyzer for GeneChip) (Affymetrix) and Partek Genomic Suite (Partek, St Louis, MO, USA) were used to validate copy number alterations. The qualities of the results were high in every sample $(250 \mathrm{~K}$ array: SNP call rate $>95 \%$, multifactor dimensionality reduction $>99 \%$, (multifactor dimensionality reduction-minimum candidate region) $<5 \%$; SNP array 6.0: contrast quality control $>2$, quality control call rate $>93 \%$, median absolute pairwise difference (MAPD) $<0.4$ ).

We also performed custom high-density oligonucleotide array-comparative genomic hybridization (CGH) analysis using custom-made $4 \times 72 \mathrm{~K}$ array (Roche NimbleGen, Madison, WI, USA) based on the manufacturer's protocol. This slide covered the genomic region of chromosome 19 between 59510019 and $61490039 \mathrm{bp}$ with 71891 probes. The average probes spacing are $20 \mathrm{bp}$, and the probes encompassing two duplication regions (60144 400-60 429000 and $60860000-61051000 \mathrm{bp}$ ) are $10 \mathrm{bp}$. DNA (500 ng) was sonicated with the condition of $10 \mathrm{~s}$, power level 1 , pulse $1 \mathrm{~s}$ and duty $50 \%$ using SONIFIRE-250D (Branson, Danbury, CT, USA). Patient's DNA was labeled with Cy3-random nonamers and patient's parent (father or mother) was labeled Cy5-random nonamers. Dyes were swapped in the combination of father/patient and mother/patient (patient's DNA with Cy5 and parent DNA with Cy3). Labeled DNA was hybridized at $42^{\circ} \mathrm{C}$ for $16-20 \mathrm{~h}$, and washed. The data analysis was carried out by NimbleScan (Roche NimbleGen) and visualized by SignalMap (Roche NimbleGen).

\section{Fluorescence in situ hybridization analysis}

Fluorescence in situ hybridization (FISH) analysis was performed on metaphase chromosomes and interphase nuclei of the patient's and parental peripheral blood leukocytes. The bacterial artificial chromosome clone, RP11-384F2, mapped to the duplicated segment was used as a probe (6037456460616811 bp at 19q13.42, UCSC Genome Browser on Human, March 2006: hg18). Bacterial artificial chromosomes were labeled with Cy3-dUTP (Invitrogen, Carlsbad, CA, USA) by Nick translation kit (Vysis, Des Plaines, IL, USA). Probe-hybridization mixtures $(16 \mu \mathrm{l})$ were mounted on chromosomes/nuclei, incubated at $37^{\circ} \mathrm{C}$ for $16-72 \mathrm{~h}$ and washed. Chromosomes and nuclei were counterstained with 4',6-diamidino-2-phenylindol containing antifade solution. Fluorescence photomicroscopy was performed under an AxioCam MR CCD fitted to Axioplan2 fluorescence microscope (Carl Zeiss, Oberkochen, Germany).

\section{Quantitative real-time PCR to confirm copy number changes}

The deletion breakpoints were analyzed using genomic DNAs by quantitative real-time qPCR with Quantifast SYBR Green PCR kit on Rotor-Gene ${ }^{\mathrm{TM}} 6200$ HRM (Corbett Life Science, Sydney, Australia). The delta-delta Ct relative quantitative method was employed according to the manufacturer's protocol. Averages of duplicates were calculated by ROTOR-GENE 6000 SERIES software (Corbett Life Science).

\section{Real-time reverse transcription-PCR}

Lymphoblastoid cell lines established from the patient and her parents, and three normal controls were grown in RPMI 1640 medium supplemented with $10 \%$ fetal bovine serum, $1 \times$ antibiotic-antimycotic (Invitrogen) and $8 \mu \mathrm{g} \mathrm{m}^{-1}$ tylosin (Sigma, St Louis, MO, USA) at $37^{\circ} \mathrm{C}$ in a $5 \% \mathrm{CO}_{2}$ incubator. Total RNA was independently extracted twice using RNeasy Plus Mini Kit (Qiagen, Valencia, CA, USA). Total RNA $(2 \mu \mathrm{g})$ was subjected to reverse transcription (RT) using PrimeScript first-strand synthesis kit with random hexamers (Takara, Ohtsu, Japan). Quantitative real-time RT-PCR was performed using TaqMan Gene expression assays (Applied Biosystems) (FAM label: Hs00174148_m1 for IL11, Hs00215284_m1 for NLRP2, Hs00603423_m1 for
NLRP9, Hs00935472_m1 for NLRP11; VIC-label: Human ACTB ( $\beta$-actin) Endogenous Control). Multiplex quantitative real-time RT-PCR was carried out by the two standard curve methods on RoterGene-6200 HRM (Corbett Life Science). Relative gene expression was calculated in comparison with that of the reference (used in standard curve) cDNA. The data from duplicated experiments using two distinctive RNA samples were averaged and the standard deviation was calculated.

\section{RESULTS}

Clinical features of the proband with de novo duplications

The proband is a 15-year-old female subject who developed s-JIA at 2 years old, with swelling of her left knee and limping. She was resistant to antibiotics, anti-inflammatory therapy, methylprednisolone pulse therapy, cyclosporin A, mizoribine, methotrexate and azathioprine. As she was resistant to all the conventional therapies, she was admitted to Yokohama City University Hospital at 9 years old and received tocilizumab (anti-IL-6 receptor antibody) therapy. After starting tocilizumab, her condition got recovered and stable since then.

\section{Whole-genome oligonucleotide SNP array}

We performed analysis of 50 patients of s-JIA using whole-genome oligonucleotide SNP array (250K NspI or SNP 6.0; Affymetrix). The total copy number abnormalities were 9 deletions and 12 duplications (Table 1).

\section{Confirmation of CNVs by FISH and qPCR}

Copy number changes were confirmed by other methods like FISH or qPCR and their origin was also checked using parental DNA if available. Most of the copy number abnormalities were of either paternal or maternal origin (Table 1), but two duplications at 19q13.42 on the patient (ID1395) occurred de novo, being confirmed by FISH analysis (Figure 1) and qPCR (data not shown).

\section{Custom array-CGH}

In addition, we performed custom oligonucleotide $72 \mathrm{~K}$ array-CGH (Roche NimbleGen), to check the precise size of the duplications. CGH was performed using the following combination of test DNA/ reference DNA: patient/father and patient/mother. Dye-swap analysis was also performed (Figure 2). As a result, two de novo microduplications at 19q13.42 with the size of $77 \mathrm{~kb}$ (Genome browser version hg18, chromosome 19 coordinates: $60190370-60267627 \mathrm{bp}$ ) and $622 \mathrm{~kb}$ (60 $377092-60991185$ bp), separated by a 109-kb segment of normal copy number (60267 627-60 377092 bp), were identified. Two sets of test DNA/reference DNA combinations consistently showed the duplications, indicating that the duplications occurred de novo. Parentage of the family was confirmed using microsatellite markers (data not shown). Similar duplication was never deposited to the DECIPHER database (http://decipher.sanger.ac.uk/syndrome), although only one overlapping deletion was found in a patient with autism.

\section{Expression analysis of duplicated genes}

IL11, NLRP2, NLRP9 and NLRP11 were chosen to see their expression as they were mapped to the duplication. Low expression of IL11 and NLRP9 in lymphoblasts hindered the proper evaluation. NLRP2 and NLRP11 did show variable expression patterns depending on lymphoblastoid cells; thus, it was indeed difficult to see the effect of duplication of these genes (data not shown).

\section{DISCUSSION}

We identified two de novo microduplications in one out of $50 \mathrm{~s}$-JIA patients. The duplications contain several interesting genes, including 
Table 1 CNVs found in S-JIA patients

\begin{tabular}{|c|c|c|c|c|c|c|c|}
\hline Patient ID & CNV overlap (\%) & Size & Deletion & Duplication & Confirmation & Inheritance & Array type \\
\hline 1239 & Partial CNV & $267 \mathrm{~kb}$ & & $\operatorname{dup}(3)(p 12.2)$ & qPCR & Not available & $250 \mathrm{~K}$ \\
\hline 1247 & 0 & $275 b$ & $\operatorname{del}(11)(q 21)$ & & FISH & Inherit (father) & $250 \mathrm{~K}$ \\
\hline \multirow[t]{2}{*}{1287} & Partial CNV & $1.58 \mathrm{mb}$ & $\operatorname{del}(2)(q 13)$ & & FISH & Inherit (mother) & $250 \mathrm{~K}$ \\
\hline & 0 & $259 \mathrm{~kb}$ & & $\operatorname{dup}(3)(\mathrm{p} 26.1)$ & $\mathrm{qPCR}$ & Inherit (mother) & \\
\hline 1317 & 0 & $13 \mathrm{~kb}$ & $\operatorname{del}(16)(q 24.1)$ & & qPCR & Not available & SNP 6.0 \\
\hline 1350 & 0 & $50 \mathrm{~kb}$ & $\operatorname{del}(4)(q 34.2)$ & & $\mathrm{qPCR}$ & Not available & SNP 6.0 \\
\hline 1361 & 0 & $128 \mathrm{~kb}$ & $\operatorname{del}(2)(q 13)$ & & qPCR, FISH & Inherit (mother) & SNP 6.0 \\
\hline \multirow[t]{2}{*}{1383} & 0 & 362 kb & & $\operatorname{dup}(2)(q 11.2)$ & qPCR, FISH & Inherit (mother) & SNP 6.0 \\
\hline & 0 & 558 kb & & $\operatorname{dup}(2)(q 11.2)$ & qPCR, FISH & Inherit (mother) & \\
\hline \multirow[t]{2}{*}{1395} & 27 & $622 \mathrm{~kb}$ & & $\operatorname{dup}(19)(q 13.42)$ & qPCR, FISH & De novo & SNP 6.0 \\
\hline & 58 & 77 kb & & $\operatorname{dup}(19)(q 13.42)$ & qPCR, FISH & De novo & \\
\hline 1439 & 62 & 906 kb & & $\operatorname{dup}(1)(q 43)$ & qPCR & Not available & SNP 6.0 \\
\hline 1620 & 0 & $695 \mathrm{~kb}$ & & $\operatorname{dup}(3)(q 26.31)$ & $\mathrm{qPCR}$ & Not available & SNP 6.0 \\
\hline 1669 & 0 & $85 \mathrm{~kb}$ & $\operatorname{del}(16)(q 24.1)$ & & qPCR & Not available & SNP 6.0 \\
\hline
\end{tabular}

Abbreviations: CNV, copy number variation; FISH, fluorescence in situ hybridization; qPCR, quantitative real-time polymerase chain reaction; S-JIA, systemic-onset juvenile idiopathic arthritis; SNP, single-nucleotide polymorphism.

CNV, overlap shows overlapping ratio of reported CNVs. Inheritance indicates parental origin of CNVs. Not available: parental samples were unavailable.

De novo change is shown in bold.
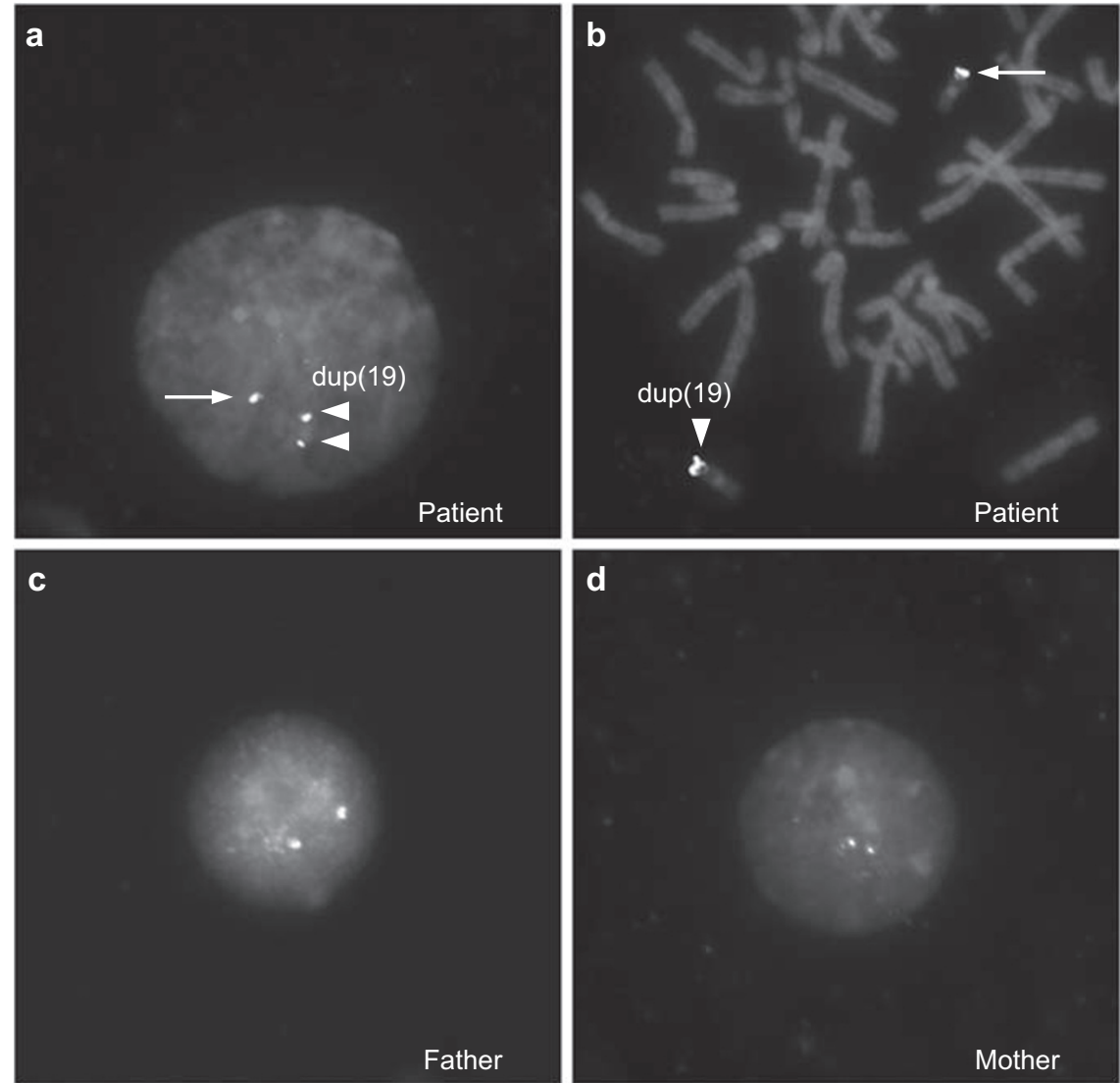

Figure 1 Fluorescence in situ hybridization (FISH) analysis using a bacterial artificial chromosome (BAC) clone, RP11-384F2, on cells of the family. (a) Interphase nucleus of the patient, (b) metaphase chromosomes of the patient and (c, d) father's and mother's interphase nuclei, respectively. Arrowhead indicates double signals showing duplication at 19q13.42 and arrow indicates a single signal of normal chromosome. 
a

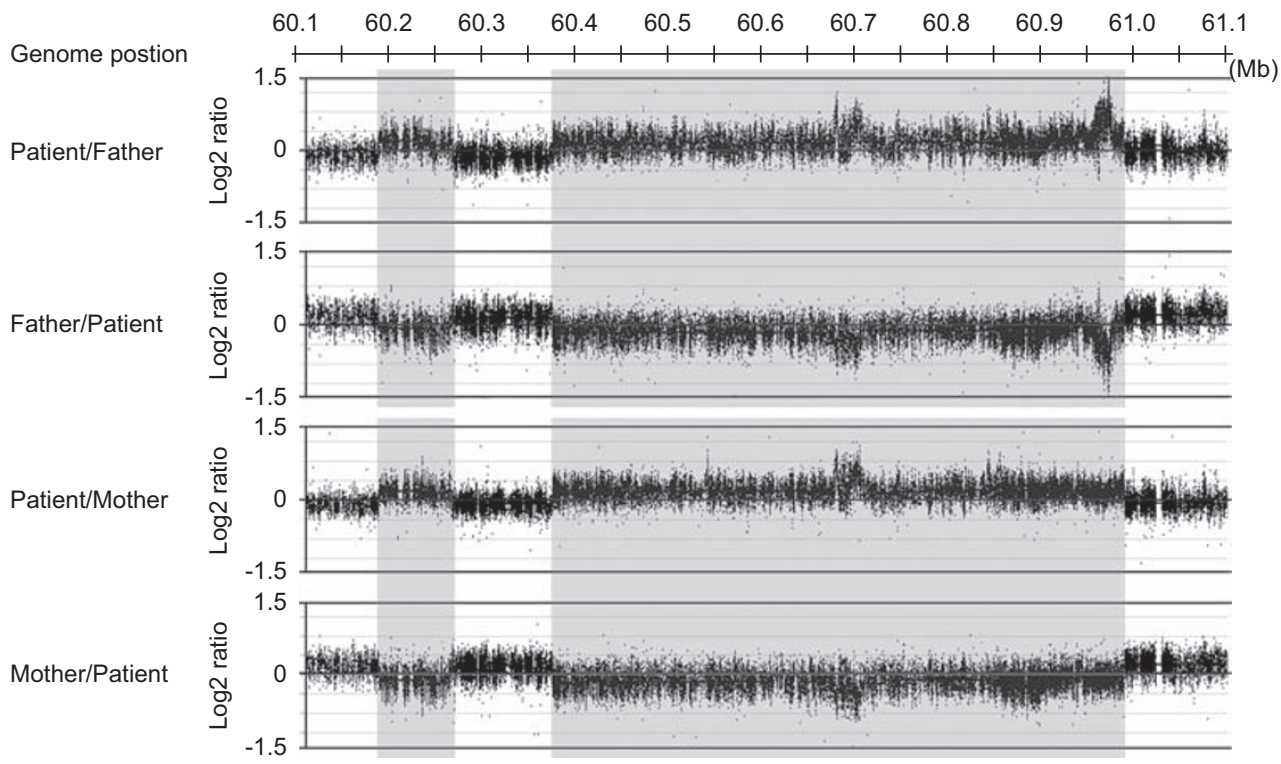

b

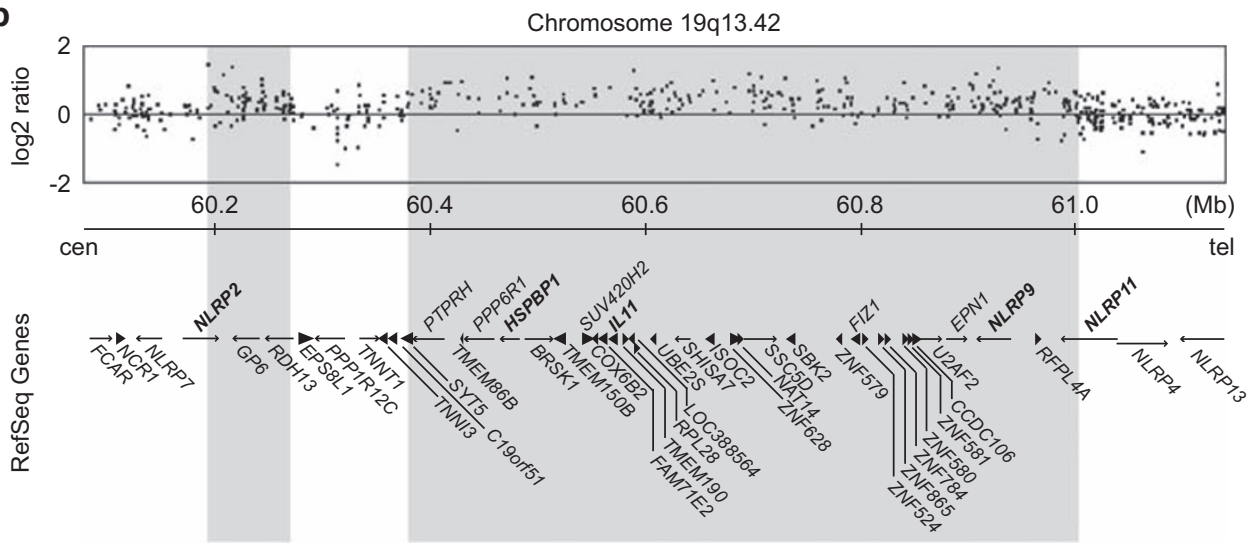

Figure 2 (a) Custom array-comparative genomic hybridization (CGH) results showing two de novo duplications at 19q13.42. Combinations of test DNA/reference DNA from top to bottom are patient/father, father/patient (dye-swapped), patient/mother and mother/patient (dye-swapped). (b) Characterization of 19q13.42 duplications. Upper panel shows the result of GeneChip Human SNP array 6.0 of chromosome 19 clearly showing duplications. Lower panel shows the RefSeq gene list at 19q13.42 corresponding to duplications. Duplications include NLRP families, IL11 and HSPBP1.

NRLP family (NLRP2, NLRP9 and NLRP11), IL11 and HSPBP1, all of which are correlated with inflammatory pathways.

The NLRP family is composed of 14 members, including NLRP2, NLRP9 and NLRP11 (ref. 10). Most NLRPs are encoded by two gene clusters on chromosome 11p15 (NLRP6, NLRP10 and NLRP14) and 19q13.4 (NLRP2, NLRP4, NLRP5, NLRP7, NLRP8, NLRP9, NLRP11, NLRP12 and NLRP13) (ref. 10). NLRPs are evolutionally conserved through Caenorhabditis elegans, Drosophila melanogaster, rat, mouse and human. NLRP1 and NLRP3 are known as components of the inflammasome implicated in early detection of extracellular pathogens and intracellular noxious compounds and driving inflammatory and immune responses. ${ }^{11}$ Germline mutations in NLRP3 and NLRP12 are associated with hereditary periodic fever syndromes. ${ }^{12,13}$ Although the function of NLRP9 and NLRP11 is not well understood, NLRP2 is suggested to function as a modulator of macrophage nuclear factor- $\kappa \mathrm{B}$ activation and caspase- 1 activation, ${ }^{14}$ which may cause inflammation. All the evidences support that duplications of the NRLP gene cluster may significantly contribute to s-JIA pathogenesis in the patient.
IL-6 and IL-11 bind to their own ligand-specific receptor (IL-6R or IL-11R) and recruit a homodimer of gp130, which is responsible for intracellular signaling. ${ }^{15}$ The gp130 signaling cytokines contribute to inflammation and bone homeostasis. IL- 6 and IL-11 play an important role in osteoclast development, such as promoting osteoclastogenesis and bone resorption in bone marrow cultures. ${ }^{16,17}$ IL-6 and IL-11 are capable of inducing osteoclast formation from peripheral blood mononuclear cells by a receptor activator of nuclear factor- $\mathrm{\kappa B}$ ligand-independent mechanism. ${ }^{18}$ IL-11 is produced by fibroblasts, mesenchyme-derived stromal cells of bone marrow, osteoblasts and chondrocytes. ${ }^{16,19}$ The expression levels of IL-11 are upregulated in patients with s-JIA compared with healthy children and positively correlated with the number of joints with active arthritis. ${ }^{20}$ Duplication of IL-11 possibly associated with its higher expression in inflammatory cells is likely to be related to the arthritis in the patient, although the roles of IL-11 are not well clarified in rheumatoid arthritis pathogenesis and alteration of IL-11 expression could not be confirmed in the patient's lymphoblastoid cells owing to the low 
expression level. It is possible that the anti-IL-6 receptor antibody therapy effectively suppressed the upregulated gb130 signaling in part by IL-11 duplication in this patient.

Heat-shock proteins (Hsps) are essential to prokaryotic and eukaryotic cellular organisms during intracellular (un)folding, assembly and translocation of proteins. ${ }^{21}$ Their synthesis is greatly enhanced in response to a variety of stressful stimuli such as temperature, hypoxia, infection and inflammation. ${ }^{22}$ Microbial Hsps are a potential inducer of crossreactive immune responses to host self-molecules that may lead to autoimmunity. ${ }^{23}$ In several experimental models, $\mathrm{T}$ cells responding to Hsps play an important role in the regulation of peripheral tolerance and suppressing pathogenic immune response. ${ }^{24}$ HspBP1 (Hsp70 binding protein 1) has an inhibitory effect in Hsp70assisted refolding reactions in the cytosol, ${ }^{25,26}$ suggesting that HspBP1 plays an important role in regulating immune responses. HspBP1 duplication may alter immune responses in the s-JIA patient.

Genomic duplications can be pathogenic through both increased dosage (whole gene duplication, duplication of regulatory elements) and disruption of coding regions (intragenic duplications). Intronic duplications potentially disrupting the splicing machinery have also been reported to be pathogenic. ${ }^{27}$ In our study, we found de novo duplications in one s-JIA patient, which involve many important genes for the regulation of the immune system. Other patients without any pathological CNVs in this study may have point mutations of a gene(s) mapped to the duplications, which may lead to upregulated gene expression causative for s-JIA as previously described in a different disease. ${ }^{28}$ Further analysis is absolutely necessary.

In conclusion, this is the first report describing a possible relationship between CNVs and s-JIA, and we believe such abnormal genotypes are important to solve the pathogenesis of s-JIA.

\section{ACKNOWLEDGEMENTS}

We thank patients and their families for their participation in this study. This work was supported by grants from the Ministry of Education, Culture, Sports, Science and Technology (NM), the Japan Science and Technology Agency (NM), the Ministry of Health, Labour and Welfare, Japan (SY and NM) and Mother and Child Health Foundation (NM).

1 Symmons, D. P., Jones, M., Osborne, J., Sills, J., Southwood, T. R. \& Woo, P. Pediatric rheumatology in the United Kingdom: data from the British Pediatric Rheumatology Group National Diagnostic Register. J. Rheumatol. 23, 1975-1980 (1996).

2 Hinks, A., Barton, A., Shephard, N., Eyre, S., Bowes, J., Cargill, M. et al. Identification of a novel susceptibility locus for juvenile idiopathic arthritis by genome-wide association analysis. Arthritis Rheum. 60, 258-263 (2009)

3 Glass, D. N. \& Giannini, E. H. Juvenile rheumatoid arthritis as a complex genetic trait. Arthritis Rheum. 42, 2261-2268 (1999).

4 Thomson, W. \& Donn, R. Juvenile idiopathic arthritis genetics-What's new? What's next? Arthritis Res. 4, 302-306 (2002).

5 Fishman, D., Faulds, G., Jeffery, R., Mohamed-Ali, V., Yudkin, J. S., Humphries, S. et al. The effect of novel polymorphisms in the interleukin-6 (IL-6) gene on IL-6 transcription and plasma IL-6 levels, and an association with systemic-onset juvenile chronic arthritis. J. Clin. Invest. 102, 1369-1376 (1998).

6 Donn, R. P., Shelley, E., Ollier, W. E. \& Thomson, W. A novel 5'-flanking region polymorphism of macrophage migration inhibitory factor is associated with systemiconset juvenile idiopathic arthritis. Arthritis Rheum. 44, 1782-1785 (2001).

7 Donn, R., Alourfi, Z., De Benedetti, F., Meazza, C., Zeggini, E., Lunt, M. et al. Mutation screening of the macrophage migration inhibitory factor gene: positive association of a functional polymorphism of macrophage migration inhibitory factor with juvenile idiopathic arthritis. Arthritis Rheum. 46, 2402-2409 (2002).

8 De Benedetti, F., Meazza, C., Vivarelli, M. Rossi, F., Pistorio, A., Lamb, R et al. Functional and prognostic relevance of the -173 polymorphism of the macrophage migration inhibitory factor gene in systemic-onset juvenile idiopathic arthritis. Arthritis Rheum. 48, 1398-1407 (2003).

9 Nannya, Y., Sanada, M., Nakazaki, K., Hosoya, N., Wang, L., Hangaishi, A. et al. A robust algorithm for copy number detection using high-density oligonucleotide single nucleotide polymorphism genotyping arrays. Cancer Res. 65, 6071-6079 (2005).

10 Tschopp, J., Martinon, F. \& Burns, K. NALPs: a novel protein family involved in inflammation. Nat. Rev. Mol. Cell. Biol. 4, 95-104 (2003).

11 Drenth, J. P. \& van der Meer, J. W. The inflammasome-a linebacker of innate defense. N. Engl. J. Med. 355, 730-732 (2006).

12 Jeru, I., Duquesnoy, P., Fernandes-Alnemri, T., Cochet, E., Yu, J. W., , Lackmy-Port-Lis, M. et al. Mutations in NALP12 cause hereditary periodic fever syndromes. Proc. Natl Acad. Sci. USA 105, 1614-1619 (2008).

13 Hoffman, H. M., Mueller J. L., Broide, D. H. Wanderer, A. A \& Kolodner, R. D. Mutation of a new gene encoding a putative pyrin-like protein causes familial cold autoinflammatory syndrome and Muckle-Wells syndrome. Nat. Genet. 29, 301-305 (2001).

14 Bruey, J. M., Bruey-Sedano, N., Newman, R., Chandler, S., Stehlik, C. \& Reed, J. C. PAN1/NALP2/PYPAF2, an inducible inflammatory mediator that regulates NF-kappaB and caspase-1 activation in macrophages. J. Biol. Chem. 279, 51897-51907 (2004).

15 Sims, N. A. \& Walsh, N. C. GP130 cytokines and bone remodelling in health and disease. BMB Rep. 43, 513-523.

16 Girasole, G., Passeri, G., Jilka, R. L. \& Manolagas, S. C. Interleukin-11: a new cytokine critical for osteoclast development. J. Clin. Invest. 93, 1516-1524 (1994).

17 Heymann, D. \& Rousselle, A. V. gp130 cytokine family and bone cells. Cytokine $\mathbf{1 2}$ $1455-1468$ (2000).

18 Kudo, O., Sabokbar, A., Pocock, A., Itonaga, I., Fujikawa, Y. \& Athanasou, N. A. Interleukin- 6 and interleukin-11 support human osteoclast formation by a RANKLindependent mechanism. Bone 32, 1-7 (2003)

19 Hill, P. A., Tumber, A., Papaioannou, S. \& Meikle, M. C. The cellular actions of interleukin-11 on bone resorption in vitro. Endocrinology 139, 1564-1572 (1998).

20 Mima, T., Ishikawa, S., Aoki, C., Yoshio-Hoshino, N., Adachi, Y., Imagawa, T. et al. Interleukin 11 and paired immunoglobulin-like type 2 receptor alpha expression correlates with the number of joints with active arthritis in systemic juvenile idiopathic arthritis. Ann. Rheum. Dis. 68, 286-287 (2009).

21 Gething, M. J. \& Sambrook, J. Protein folding in the cell. Nature 355, 33-45 (1992).

22 Welch, W. J., Kang, H. S., Beckmann, R. P. \& Mizzen, L. A. Response of mammalian cells to metabolic stress; changes in cell physiology and structure/function of stress proteins. Curr. Top. Microbiol. Immunol. 167, 31-55 (1991).

23 Albani, S. \& Carson, D. A. A multistep molecular mimicry hypothesis for the pathogenesis of rheumatoid arthritis. Immunol. Today 17, 466-470 (1996).

24 van Eden, W., van der Zee, R., Paul, A. G., Prakken, B. J., Wendling, U., Anderton, S. M. et al. Do heat shock proteins control the balance of T-cell regulation in inflammatory diseases? Immunol. Today 19, 303-307 (1998).

25 Shomura, Y., Dragovic, Z., Chang, H. C., Tzvetkov, N., Young, J. C., Brodsky, J. L. et al. Regulation of Hsp70 function by HspBP1: structural analysis reveals an alternate mechanism for Hsp70 nucleotide exchange. Mol. Cell 17, 367-379 (2005).

26 Raynes, D. A. \& Guerriero, V. Jr Inhibition of Hsp70 ATPase activity and protein renaturation by a novel Hsp70-binding protein. J. Biol. Chem. 273, 32883-32888 (1998).

27 Bovolenta, M., Neri, M., Fini, S., Fabris, M., Trabanelli, C., Venturoli, A. et al. A novel custom high density-comparative genomic hybridization array detects common rearrangements as well as deep intronic mutations in dystrophinopathies. BMC Genom. 9, 572 (2008)

28 Gimelli, S., Caridi, G., Beri, S., McCracken, K., Bocciardi, R., Zordan, P. et al. Mutations in SOX17 are associated with congenital anomalies of the kidney and the urinary tract. Hum. Mutat. 31, 1352-1359. 